\title{
AN APPROACH TO THE CLASSIFICATION OF UNKNOWN BIOLOGICAL AGENTS WITH CELL BASED SENSORS
}

\author{
David A. Borkholder, B. Derek DeBusschere, and Gregory T.A. Kovacs \\ Electrical Engineering, Stanford University \\ Center for Integrated Systems, CISX 202X, Stanford, CA 94305-4075
}

\begin{abstract}
The broad-spectrum sensitivity of cell based biosensors offers the capability for detecting previously unknown biological agents. One cellular parameter that is often measured is the action potential of electrically active cells. However, the complexity of this signal makes interpretation of the cellular response to a compound difficult to interpret. By analyzing shifts in the signal's power spectrum, it may be possible to classify the ionic channels modulated by the agent.

A system is described for the measurement of action potentials from cells cultured on a planar microelectrode array. Experimental results, simulations, and analyses are presented for three pharmaceuticals tested on chick myocardial cells. While the actual agents could readily be distinguished experimentally, the models used for simulation were a partial success, accurately predicting the response to one of the three agents tested.
\end{abstract}

\section{INTRODUCTION}

Cell based sensor technologies utilizing electrically active living cells cultured on extracellular electrode arrays have been a focus of research since 1972 [1]. However, most efforts have been directed towards pure neuroscience applications that require experienced interpretation of the signals. More recent work by some groups has attempted to expand the practical uses of action potential (AP) based systems to environmental and chemical monitoring and pharmaceutical screening [2-5]. However, there has been little work done on signal interpretation models for general classification of agents modulating cellular electrical characteristics. This work attempts to classify the type of ion channel $\left(\mathrm{Na}^{+}, \mathrm{K}^{+}, \mathrm{Ca}^{2+}\right)$ affected by an unknown, biologically active agent.

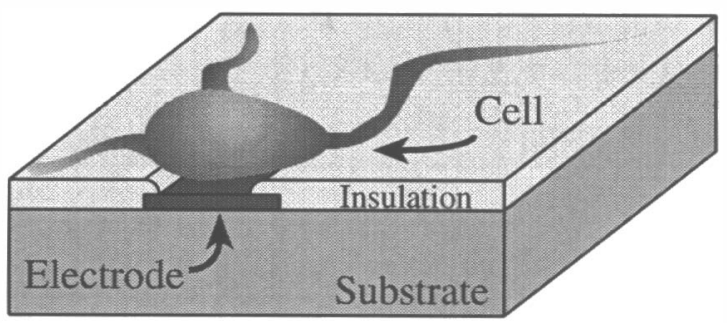

Figure 1. Conceptual drawing of a cultured cell coupled to a microelectrode.

Electrical activity in cells is generated by a complex interaction of both ligand and voltage gated ion channels. As a cell depolarizes (transmembrane potential changes), the voltage threshold for opening of different ion channels is reached, resulting in electrochemical gradient driven flow of ions through the cell membrane. Different channel classes open at different times and contribute to unique portions of this action potential. The cardiac AP studied in this work can be divided into several phases: an initial rapid depolarization due to increased $\mathrm{Na}^{+}$conductance; a plateau phase due to the slow opening of voltage-gated $\mathrm{Ca}^{2+}$ channels; and the final repolarization due to the closure of $\mathrm{Ca}^{2+}$ channels and the prolonged opening of $\mathrm{K}^{+}$channels [6]. The authors postulate that the frequency content of an AP may be correlated with specific ion channel classes. As the normal operation of these channels is altered by chemical or biological agents (toxins, pharmaceuticals, etc.), it is expected that the power spectral density (PSD) of the AP will change in a characteristic way for each channel type. Determination of a unique modulation function of a pharmaceutical on the AP may provide a useful method for rapid characterization of channel modulating agents.

\section{MICROELECTRODE ARRAY}

The microelectrodes used in this research consist of a $6 \times 6$ array of circular iridium, gold or platinum electrodes of varying areas $\left(15\right.$ to $\left.480 \mu \mathrm{m}^{2}\right)$. The electrodes are fabricated on either glass or silicon substrates and have the basic structure shown in Figure 1. The dice are packaged in a ceramic 40 pin dual in-line package (DIP), and bond-wire connections are made between the die and package. The bond-wires are encapsulated in a biocompatible epoxy that is also used to attach a polystyrene petri dish to form a cell culture chamber as shown in Figure 2. Electrode impedances are reduced by electroplating platinum black on the electrode surface and using ultrasonic agitation to ensure adequate adhesion [7]. The fabrication, packaging and platinizing procedures have been described in detail elsewhere [8]. The packaged microelectrode arrays are used as a substrate for the culture of embryonic chick myocardial cells. These cells form a spontaneously beating syncytium which generates APs that travel through the cell layer. The electrical signals are monitored using the extracellular microelectrodes and low noise amplifiers.

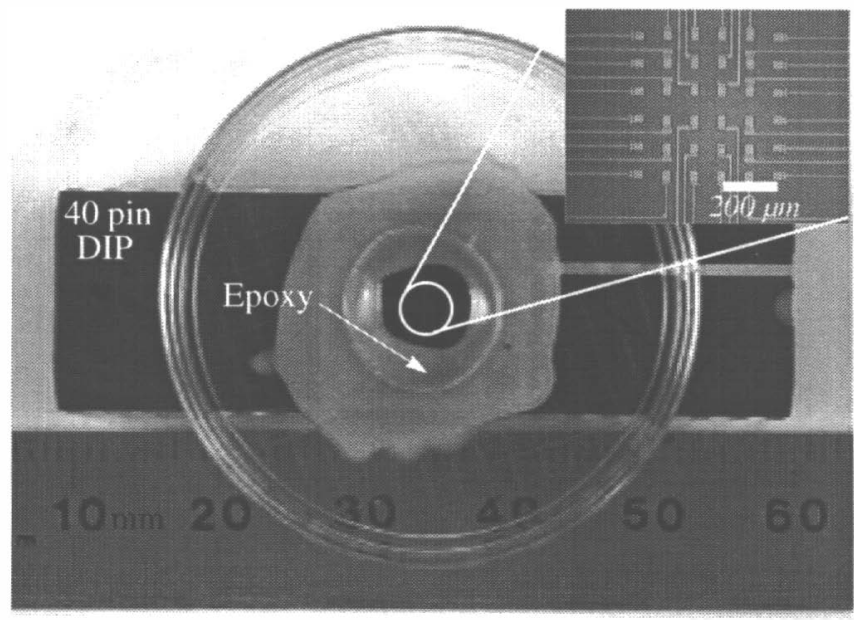

Figure 2. A microelectrode array is packaged in a ceramic 40 pin DIP. Bond-wires are encapsulated in epoxy and a $35 \mathrm{~mm}$ polystyrene cell culture dish (with hole drilled in the center) is attached to the top. Inset shows a close-up of the $6 \times 6$ element electrode array. 


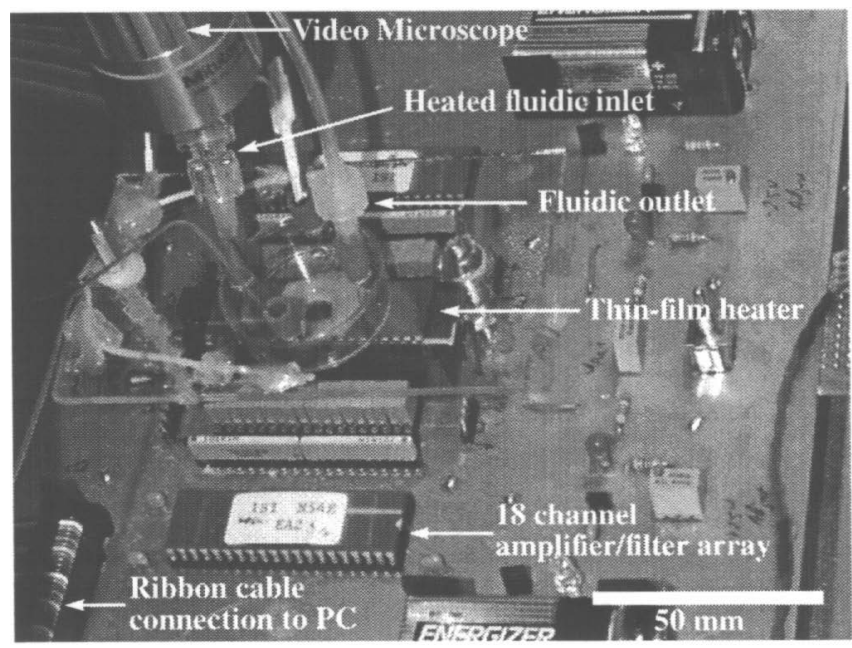

Figure 3. Measurement setup used for AP monitoring. The cell culture chamber is connected to the electronics via a ZIF socket. The amplified action potentials (through the custom 18 channel amplifier/filter arrays) are measured by direct connection to a PC via ribbon cables.

\section{MEASUREMENT SYSTEM}

Measurement of electrical activity from cultured cells in vitro requires a low noise amplification system coupled to data acquisition and storage equipment. As shown in Figure 3, the packaged microelectrode array is placed into the measurement system using a zero insertion force (ZIF) socket. Individual electrodes are AC coupled to a custom 18 channel amplifier/filter array (developed at Stanford University [9-10]). The amplifier has a midband gain of $45 \mathrm{~dB}$, input referred noise of $8 \mu \mathrm{V}_{\text {rms }}(10 \mathrm{~Hz}$ to $10 \mathrm{kHz}$ ), and an adjustable high pass filter ( $3 \mathrm{~Hz}$ to $10 \mathrm{kHz}$ ).

The amplified AP signals are routed via ribbon cable to a PCbased data acquisition system capable of simultaneously monitoring up to 32 channels and storing the digital, 12-bit data to disk (Experimenter's Workbench 32 by DataWave Technologies, Colorado). Off-line analysis of the AP waveforms was performed using additional software tools.

In addition to the AP electronics, the measurement system must also include fluidics for sample introduction and temperature regulation. As shown in Figure 3, an indium-tin oxide (ITO) coated glass plate is positioned over the cell culture chamber. Electrical current flowing through the resistive ITO heats the glass to assist in temperature control and reduce condensation (to allow for visual observation of the cells). Holes drilled through the plate allow for insertion of a teflon coated thermistor probe, fluidic inlet and outlet tubes, and a platinum wire to be used as a grounded electrode in the solution. Fluid is heated to $37^{\circ} \mathrm{C}$ prior to entering the dish using a resistive heater surrounding the inlet tube. The fluidic outlet consists of a 30 gauge needle connected to a vacuum source adjusted to match the inlet flow rate $(2.0 \mathrm{ml} / \mathrm{min})$. Die and package temperature is controlled by a thin-film heater positioned below the DIP. The combination of the heated glass plate, inlet fluid heating, and package heating result in a temperature at the electrode array of $37^{\circ} \mathrm{C} \pm 0.3^{\circ} \mathrm{C}$.

Media flow, controlled by both gravity and tubing resistance, was adjusted to $2.0 \mathrm{ml} / \mathrm{min}$. Manual sample selection was accomplished via a teflon 8-way valve configured to allow up to four different solutions to be used in the same experiment. HEPES buffered recording media was prepared for experiments at

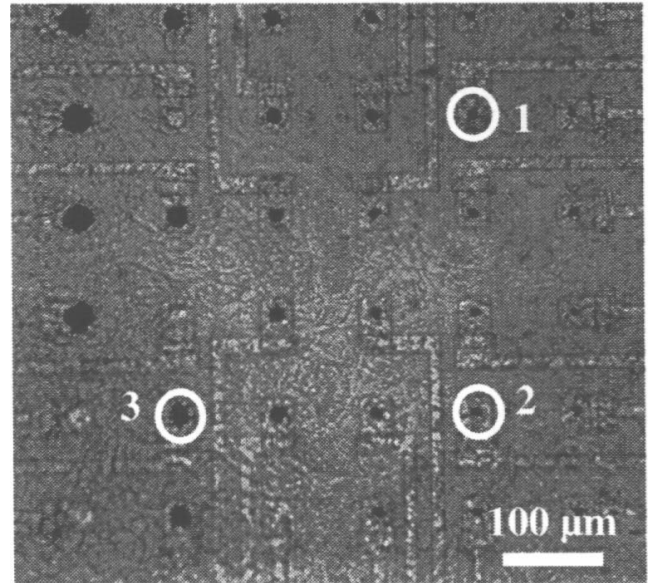

(a)

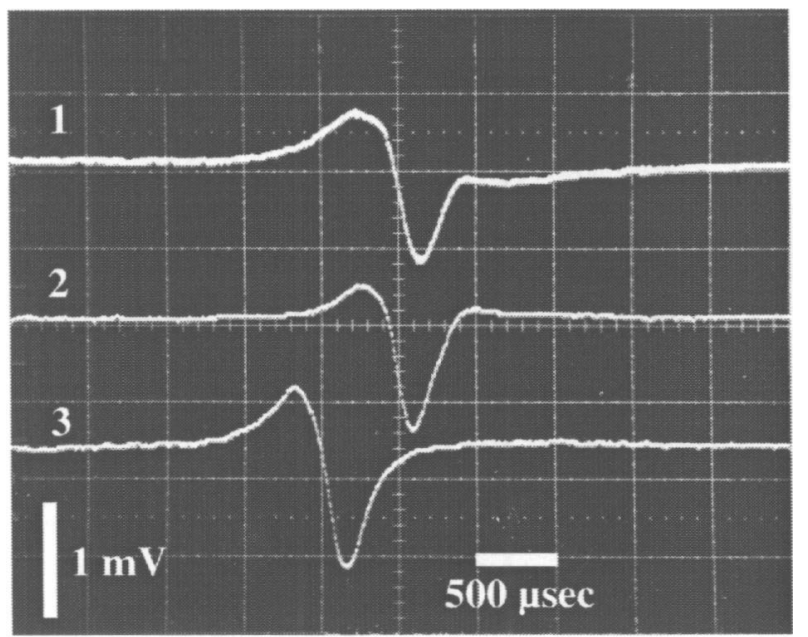

(b)

Figure 4. (a) Optical view of embryonic chick myocardial cells cultured on the microelectrode array. Spontaneous APs recorded extracellularly from the circled microelectrodes are shown in (b). The time delay observed between action potentials is due to the finite propagation velocity of the traveling action potential through the two dimensional sheet of cells.

atmospheric $\mathrm{CO}_{2}$ levels, with both the $\mathrm{pH}$ and osmolality adjusted to physiologic levels ( 7.3 and $300 \mathrm{mOsm} / \mathrm{kg}$ respectively). Where pharmaceuticals or toxins were to be tested, they were added directly to this recording media in concentrations which did not significantly alter the osmolality or $\mathrm{pH}$ of the solutions. Continuous flow was used to limit variations due to changing flow velocities and temperature which can be problematic with manual bolus injection systems.

\section{ACTION POTENTIAL MEASUREMENT AND SIMULATION}

Embryonic chick myocardial cells were explanted from 11 day old chicks and plated onto the microelectrode arrays described above at a density of 1,000 cells $/ \mathrm{mm}^{2}$ using the techniques of De Hann, Barry and Polinger [11-13]. APs were measured from the spontaneously beating, two-dimensional sheet of cells two to four days after plating. An optical image of cells cultured on the microelectrode array and typical action potential recordings are shown in Figure 4. Note the time delay between AP peaks, corresponding to the finite propagation velocity of the action potential wavefront through the tissue. 


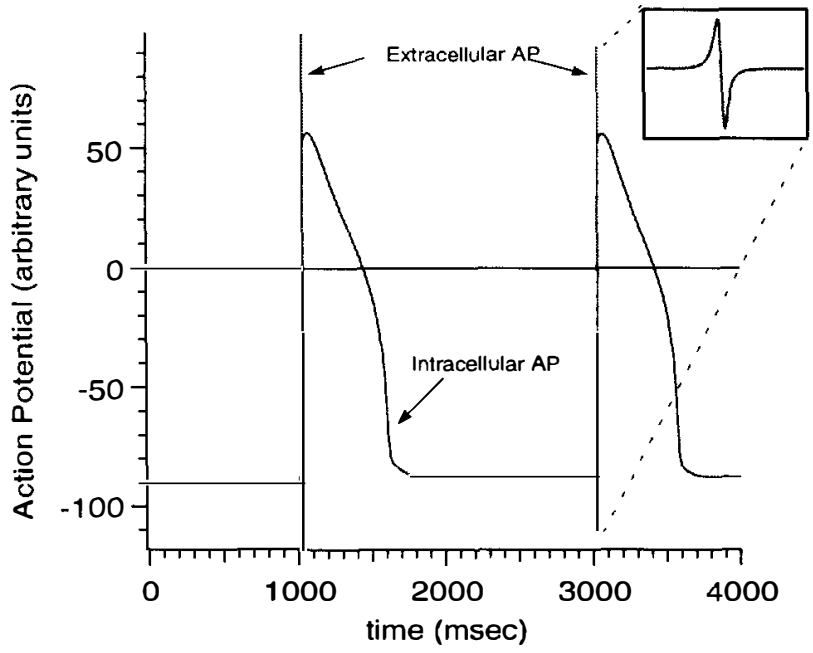

Figure 5. Simulated action potentials. Intracellular signals were calculated using SPICE and Hodgkin-Huxley type equations for the cellular membrane. The second time derivative of this intracellular signal was computed to estimate the expected extracellular signal to be recorded by a microelectrode. An expanded view of the $3 \mathrm{msec}$ wide second derivative signal is shown in the inset.

Correlation of these extracellular signals to classical intracellular recordings (large literature base) is often difficult since they represent a temporal derivative of the intracellular signal dependent on the coupling of the cell to the microelectrode. For tightly coupled cells, the extracellular signal looks like the first derivative of the intracellular. As the coupling is reduced, higher order derivatives are observed [14]. For the data presented herein, second order derivative characteristics have been confirmed by comparison to the time derivative of intracellular action potentials from cultured embryonic chick myocardial cells taken from the literature [15]. This information is important for comparison of experimental data to simulations.

Simulation of spontaneous action potentials was carried out with the circuit simulator HSPICE using Hodgkin-Huxley type models developed for bullfrog atrial cells [16]. While differences between the chick and bullfrog are likely, the availability of suitable models was extremely limited. Additionally, the precise composition of the chick cardiac cell population in culture is not known (some combination of atrial, ventricular, and sinoatrial node cell types), making exact modeling difficult. In this system, the cell membrane was modeled as a capacitance connected in parallel to a number of conductive ionic channels. The model was modified to include two cells connected by a resistive gap junction to simulate the normal electrical connections between cardiac cells. One cell was stimulated using a current pulse, firing an AP which in turn elicited an AP in the second cell. Simulated results were obtained from this second action potential and compared to those obtained experimentally.

The simulation parameters were slightly modified to provide an intracellular signal similar in shape and duration to those published for embryonic chick myocardial cells in vitro [15]. The second time derivative was then taken resulting in the waveforms of Figure 5. Note the shape and duration are similar to the actual action potentials recorded in Figure 4.

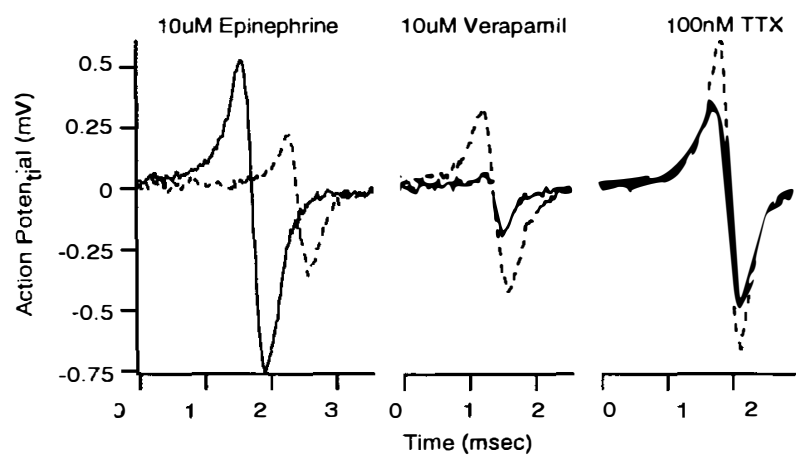

Figure 6. Action potential changes with pharmaceutical additions. Unmodulated APs are shown with dashed lines while post-agent $A P s$ are represented by solid lines. Epinephrine $\left(\mathrm{Ca}^{2+}\right.$ channel agonist), Verapamil $\left(\mathrm{Ca}^{2+}\right.$ channel blocker $)$, and Tetrodotoxin (TTX) (Fast-Na ${ }^{+}$channel blocker) effects are shown. The waveforms presented are extracellular recordings from $a$ spontaneously beating culture of embryonic chick myocardial cells cultured on a planar microelectrode array. They represent the second temporal derivative of the intracellular signal. Note: the relative timing of each $A P$ is not significant and can be altered by triggering during data acquisition.

\section{PHARMACEUTICAL MODULATION}

As mentioned previously, action potentials are the result of a complex coordination of ionic flows through the cellular membrane. The flow of specific ions is controlled by voltage or ligand gating of ion channels which exhibit specificity in ion transport. The biological effect of many toxins and pharmaceuticals is often coupled to specific binding of the agent to receptors on the cell membrane. This can result in enhanced or inhibited opening of channels or direct blockage of the ion transport region. Thus, the relative impact of the different ions on the action potential can be modulated, resulting in changes in AP shape.

Three different pharmaceuticals and toxins were examined in this study: tetrodotoxin, epinephrine, and verapamil. Tetrodotoxin (TTX) is a naturally occurring neurotoxin which binds specifically to voltage gated $\mathrm{Na}^{+}$channels. It does not affect $\mathrm{Ca}^{2+}$ or $\mathrm{K}^{+}$ channels, nor the $\mathrm{Na}^{+}$leakage that generally occurs through the

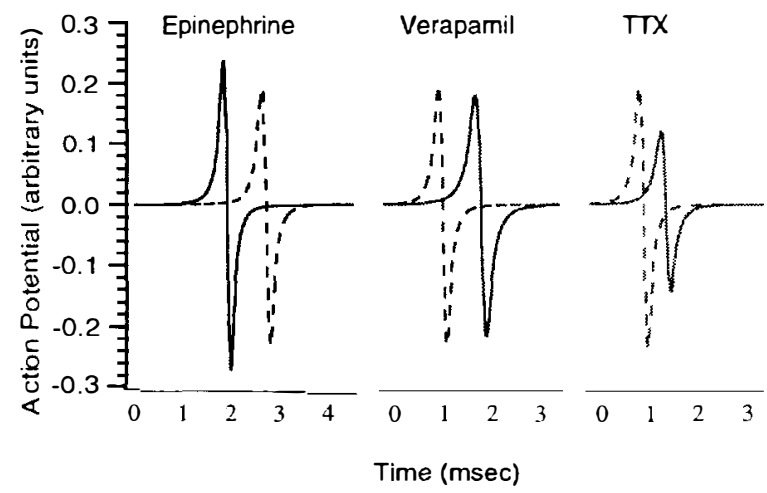

Figure 7. Simulated extracellular action potentials before and after pharmaceutical and toxin additions. Unmodulated APs are shown with dashed lines while post-agent APs are represented by solid lines. Note the responses to epinephrine and verapamil are significantly smaller than the physiological results shown in Figure 6. The response to TTX is a close approximation to that observed experimentally: Unlike Figure 6, the AP alignment offsets represent real delays rather than triggering during data acquisition. 
non-gated channels and ion pumps of the resting cell membrane [17]. The action of TTX may be easily modeled by adjustment of a scaling factor on the fast sodium current $\left(\mathrm{I}_{\mathrm{Na}}\right)$.

Epinephrine is a natural hormone released by the adrenal gland which acts on both $\alpha$ - and $\beta$-adrenergic receptors. In cardiac tissue, this agonist acts mainly on $\beta$-adrenergic receptors causing modulation of several ionic currents through second messenger pathways (cyclic adenosine 3',5'-monophosphate (cAMP) mediated). Both the inward, long-lasting (L-type) $\mathrm{Ca}^{2+}$ current $\left(\mathrm{I}_{\mathrm{Ca}, \mathrm{L}}\right)$ and the outward, delayed rectifier-type $\mathrm{K}^{+}$current $\left(\mathrm{I}_{\mathrm{K}}\right)$ are enhanced [18].

Verapamil is a lipid-soluble compound which preferentially blocks L-type $\mathrm{Ca}^{2+}$ channels. It not only reduces the magnitude of the $\mathrm{Ca}^{2+}$ current, but also decreases the rate of recovery of the channel [19]. When applied at high enough doses (tens of $\mu \mathrm{M}$ ), verapamil can also affect voltage-gated $\mathrm{K}^{+}$channels resulting in enhanced inactivation of the rectifying $I_{K}$ current [20].

From the actual data shown in Figure 6, it is clear that the compounds tested had a significant and distinct impact on the action potential shape. To simulate the effect of the pharmaceuticals and toxins, each ion channel current in the model was multiplied by a gating variable representing the percentage of channels which could open in response to an appropriate transmembrane voltage. For each agent examined, only the variable for the channel known to be affected was altered. This gating variable was adjusted to match the simulated waveforms as closely as possible to those measured experimentally. For tetrodotoxin this was achieved by blocking the fast $\mathrm{Na}^{+}$conductance by $25 \%$, which resulted in an AP amplitude reduction similar to that observed experimentally. This can be seen by a comparison of the waveforms in Figures 6 and 7 . The results for pharmaceuticals targeting calcium and potassium were not as convincing however. The effects of epinephrine could only be observed when the L-type $\mathrm{Ca}^{2+}$ and rectifying $\mathrm{K}^{+}$currents were increased by a factor of 10 (data shown in Figure 7). Even at this level, the AP amplitude was not increased as significantly as experimentally observed. Reduction of the L-type $\mathrm{Ca}^{2+}$ and rectifying $\mathrm{K}^{+}$currents by $50 \%$ to simulate the effects of verapamil resulted in only small changes in action potential amplitude. The width of the AP was modulated however, as can be seen from Figure 7.

It is important to note that both epinephrine and verapamil tend to affect the plateau phase of the action potential. Since this is not explicitly represented in the second-derivative signals obtained using extracellular microelectrodes in these experiments, the effects of pharmaceuticals acting on these channels were expected to be more difficult to observe than changes in the fast $\mathrm{Na}^{+}$conductance.

\section{POWER SPECTRAL DENSITY ANALYSIS}

To examine how the spectral characteristics of the action potentials change, the power spectral density (PSD) of each waveform was taken both before and after addition of the biologically active compound. This PSD was estimated by first taking the fast fourier transform (FFT) of the waveform, multiplying the FFT result by its complex conjugate and dividing by the frequency range. This was done for waveforms both before and after addition of the agent of interest (for simulation and experimental results). A modulation function for the pharmaceutical was then determined by dividing the PSD after agent addition by the PSD before agent addition. This ratio has been plotted for the experimental results in Figure 8 and the simulated results in Figure 9.

As can be seen from the experimental results of Figure 8,

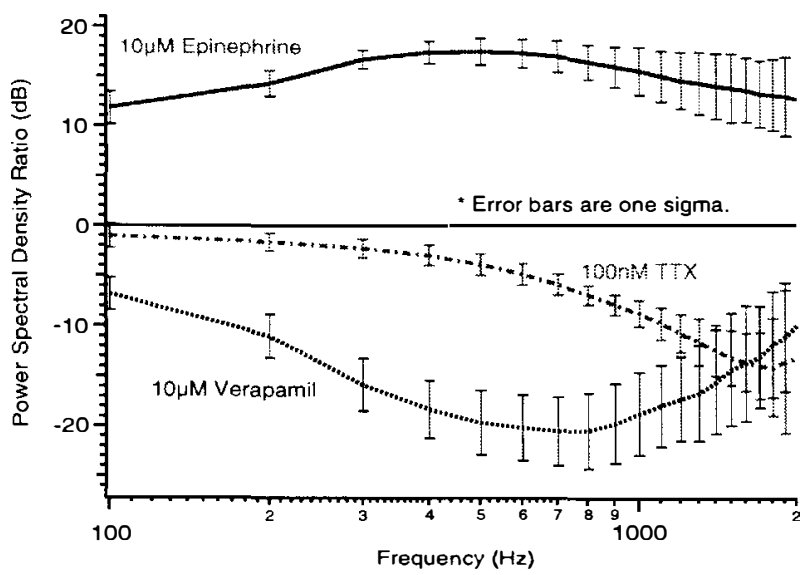

Figure 8. Power spectral density (PSD) ratio plots $\left(P S D_{\text {after agent }} \div P S D_{\text {before }}\right.$ agent $)$. Additions of $10 \mu \mathrm{m}$ epinephrine ( $\mathrm{Ca}^{2+}$ channel agonist), $100 \mathrm{nM}$ tetrodotoxin (TTX) $\left(\mathrm{Na}^{+}\right.$channel blocker), and $10 \mu \mathrm{M}$ verapamil $\left(\mathrm{Ca}^{2+}\right.$ channel blocker) are all shown to have different effects on the PSD of the measured action potential. Epinephrine and verapamil have opposite effects as expected. 8 channels of data were averaged with error bars of one sigma.

there were characteristic modulation functions observed for each of the compounds tested. Epinephrine enhanced the power of the AP across a $2 \mathrm{kHz}$ bandwidth with a slight bandpass effect observed. Verapamil had the opposite effect, reducing the overall AP power, again with a bandpass signature. Tetrodotoxin exhibited a low pass filter effect that clearly reduced the high frequency components of the AP. Additional data using a fast and slow $\mathrm{Na}^{+}$channel blocker (QX-314) resulted in a modulation function similar to that of TTX, but with additional attenuation at lower frequencies (data not shown). While extensive tests using multiple agents known to modulate similar channels have not been done, these results do show definite, unique characteristic responses for the agents tested. The similarity between the modulation functions for TTX and QX-

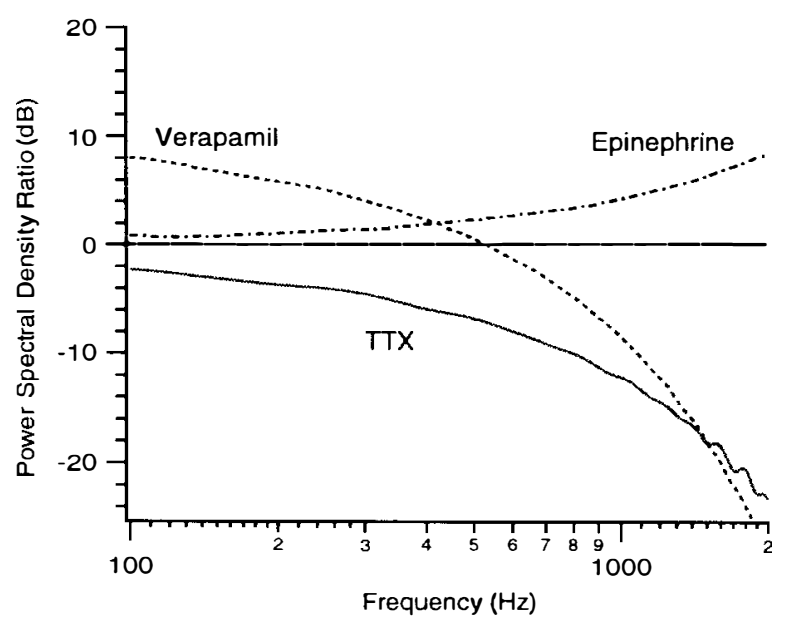

Figure 9: Simulated power spectral density (PSD) ratio plots $\left(P S D_{\text {after agent }} \div P S D_{\text {hefore agent }}\right)$. Shown are a $10 \times$ enhancement of L-type $\mathrm{Ca}^{2+}$ and rectifying $\mathrm{K}^{+}$currents for epinephrine, a $50 \%$ reduction in L-type $\mathrm{Ca}^{2+}$ and rectifying $\mathrm{K}^{+}$currents for verapamil, and a $25 \%$ reduction in $\mathrm{Na}^{+}$conductance for tetrodotoxin (TTX). The TTX simulation matches the experimental data in Figure 9 well, while those for epinephrine and verapamil were significantly different. 
314 is further evidence that unique response curves may exist for different classes of ionic channels. Further experimentation is required to verify this.

The simulated results matched extremely well for the case of tetrodotoxin, exhibiting the same lowpass response of approximately the same magnitude. However, the low frequency response of epinephrine was significantly lower than that observed experimentally, and the general shape of verapamil was completely wrong. This was most likely due to deficiencies in the empirically determined model [16] which does not accurately predict the behavior of second-messenger modulated effects, nor the subtle changes in the rising edge of the AP in response to changes in $\mathrm{Ca}^{2+}$ flux. As mentioned above, this was exacerbated by the fact that the second derivative does not represent the plateau phase of the AP, making it difficult to see effects not modeled precisely. Clearly from the experimental data of Figures 6 and 8 the pharmaceuticals tested do impact the shape and power spectrum of the extracellular action potential, but more detailed modeling is required to accurately predict these effects.

\section{SUMMARY}

The effects of three different biologically active agents (tetrodotoxin, verapamil and epinephrine) on the action potentials of spontaneously beating chick myocytes cultured on planar microelectrode arrays have been examined. The power spectral density of these APs revealed possibly unique modulation functions associated with action on specific classes of ion channels. Simulated action potential and PSD results matched those for tetrodotoxin well as expected given the second derivative nature of the measured signals. Simulation of the effects of verapamil and epinephrine resulted in modulation function shapes significantly different from those observed experimentally. This was attributed to deficiencies in the model.

While further simulation and experimental work are required to confirm the utility of the technique, the use of power spectral density analysis for classification of an unknown agent modulating ionic channels appears promising. A real-time version of such a technique could be implemented using simple analog/digital hardware, making its use in sensor applications and pharmaceutical screening more practical.

\section{ACKNOWLEDGMENTS}

The authors would like to thank Dr. Marco Bove and Dr. Joseph Pancrazio for their invaluable and greatly appreciated assistance with the action potential simulations, Dr. Nadim Maluf for his insights on data interpretation, and Dr. David Stenger for his continued support and guidance.

Funding for this project was provided by the DARPA MicroFlumes Program (Contract Number: N66001-96-C-8631).

\section{REFERENCES}

1. Thomas, C.A., Springer, P.A., Loeb, G.E., Berwald-Netter, Y. and Okun, L.M., "A miniature microelectrode array to monitor the bioelectric activity of cultured cells", Experimental Cell Research, 74:61-66 (1972).

2. Gross, G.W., Rhoades, B.K., Azzazy, H.M.E. and Wu, M.C., "The use of neuronal networks on multielectrode arrays as biosensors", Biosensors and Bioelectronics, 10:553-567 (1995).

3. Hickman, J.J., Foster, K.E., Kowtha, V., Bey, P. Jr. and Stenger, D.A., "Whole cell based biosensors", Abstracts of Papers American Chemical Society', 207(1-2):BTEC 76 (1994).
4. Stenger, D.A. and McKenna, T.M. (Eds.), Enabling technologies for cultured neural networks, Academic Press, San Diego, CA (1994).

5. Israel, D.A., Edell, D.J. and Mark, R.G., "Time delays in propagation of cardiac action potential", American Journal of Physiology: Heart and Circulatory Physiology, 27(6):H1906-H1917 (1990).

6. Ganong, W.F., Review of medical physiology, Prentice Hall, New Jersey (1993).

7. Marrese, C.A., "Preparation of strongly adherent platinum black coatings", Analytical Chemistry, 59:217-218 (1987).

8. Borkholder, D.A., Bao, J., Maluf, N.I., Perl, E.R. and Kovacs, G.T.A., "Microelectrode arrays for stimulation of neural slice preparations”, Journal of Neuroscience Methods, 77:61-66 (1997).

9. Kewley, D.T., Hills, M.D., Borkholder, D.A., Opris, I.E., Maluf, N.I., Storment, C.W., Bower, J.M. and Kovacs, G.T.A., "Plasma-etched neural probes", Sensors and Actuators: A Physical, 58:27-35 (1997).

10. Borkholder, D.A., Opris, I.E., Maluf, N.I. and Kovacs, G.T.A., "Planar electrode array systems for neural recording and impedance measurements", Conference Proceedings of the 1996 IEEE Engineering in Medicine and Biology, Amsterdam, The Netherlands, 10/31-11/3, IEEE (1996).

11. De Hann, R.L., "Regulation of spontaneous activity and growth of embryonic chick heart cells in tissue culture", Developmental Biology, 16:216-249 (1967).

12. Barry, W.H., Pitzen, R., Protas, K. and Harrison, D.C., "Inotropic effects of different calcium ion concentrations on the embryonic chick ventricle", Circulation Research, 36:727-734 (1975).

13. Polinger, I.S., "Separation of Cell Types in Embryonic Heart Cell Cultures”, Experimental Cell Research, 63:78-82 (1970).

14. Grattarola, M. and Martinoia, S., "Modeling the neuron-microtransducer junction: from extracellular to patch recording", IEEE Transactions on Biomedical Engineering, 40(1):35-41 (1993).

15. Connolly, P., Clark, P., Curtis, A.S.G., Dow, J.A.T. and Wilkinson, C.D.W., "An extracellular microelectrode array for monitoring electrogenic cells in culture", Biosensors and Bioelectronics, 5:223-234 (1990).

16. Rasmusson, R.L., Clark, J.W., Giles, W.R., Robinson, K., Clark, R.B., Shibata, E.F. and Campbell, D.L., "A mathematical model of electrophysiological activity in a bullfrog atrial cell", American Journal of Physiology, 259(2):H370-H389 (1990).

17. Hille, B., lonic channels of excitable membranes, Second edition, Sinauer Associates, Inc., Sunderland, Massachusetts (1992).

18. Kandel, E.R., Schwartz, J.H. and Jessell, T.M. (Eds.), Principles of neural science, Third edition, Appleton and Lange, Norwalk, Connecticut (1991).

19. Hardman, J.G. and Limbird, L.E. (Eds), Goodman \& Gilman's The pharmacological basis of therapeutics, Ninth edition, McGraw-Hill, New York (1996).

20. Pancrazio, J.J., Viglione, M.P., Kleiman, R.J. and Kim, Y.I. "Verapamil-induced blockade of voltage-activated potassium ion current in small-cell lung cancer cells" Journal Of Pharmacology And Experimental Therapeutics, 257(1):184-191 (1991). 